\title{
O AGEÍSMO NOS CUIDADOS DE SAÚDE: UMA REVISÃO SISTEMÁTICA
}

\author{
Sheyla Fernandes ${ }^{1}$, Nycolas Emanuel Tavares de Lira² e \\ Alanda Maria Ferro ${ }^{3}$ \\ 1,2,3Universidade Federal de Alagoas, Brasil.sheyla.fernandes@ip.ufal.br, nycolas.tavares@hotmail.com \\ maria@outlook.com
}

\begin{abstract}
Resumo. Em meio ao crescente envelhecimento natural da população mundial e a falta de distinção entre o processo natural e o patológico do envelhecer surge o ageísmo. O objetivo deste estudo é analisar a expressão do ageísmo nos cuidados em saúde, bem como vem sendo investigado pela literatura e seu desfecho para à saúde do idoso. Os dados foram buscados nas bases: Scielo, Lilacs, PsicINFO, Scopus, Web of Science e Pubmed com a chave de busca: "ageismo AND idoso AND Assistência à saúde" e "ageism AND aged AND healthcare". De 342 artigos, 64 foram selecionados, sendo extraídas as introduções e conclusões dos estudos e criados dois corpus textuais, analisados pela Classificação Hierárquica Descendente (CHD) do software Iramuteq. O primeiro corpus indicou 2 classes, a primeira ligada ao conceito de ageísmo e ocorrência no ambiente de saúde. A segunda, relacionou-se com as formas de investigação desse preconceito. O segundo corpus também resultou em duas, a primeira relacionada às sugestões para novos estudos, a segunda apresentou os aspectos negativos. Diante do exposto, é evidente a presença do ageísmo e suas consequências à saúde dos idosos. Por fim, os estudos apontam estratégias educacionais sobre o ageísmo e envelhecimento como forma de combater esse preconceito.
\end{abstract}

Palavras-chave: Ageísmo; Assistência à Saúde; Envelhecimento; Idoso; Preconceito.

\section{AGEISM IN HEALTH CARE: A SYSTEMATIC REVIEW}

Abstract. Amidst the growing natural aging the world population and the lack of distinction between the natural and pathological processes of aging, ageism arises. The aim this study is to analyze the expression of ageism in health care, as well as being investigated in the literature and its outcome for health of the elderly. The data were searched in the bases: Scielo, Lilacs, PsicINFO, Scopus, Web of Science and Pubmed with the search key: "ageism AND aged AND healthcare" and "ageism AND aged AND healthcare". Of 342 articles, 64 were selected, the introductions and conclusions the studies were extracted and two textual corpus were created, analyzed by the Descending Hierarchical Classification (CHD) of the Iramuteq software. The corpus indicated 2 classes, the first linked to the concept ageism and occurrence in the health environment. The second was related to the forms of investigation of this prejudice. The second corpus also resulted in two, the first related to suggestions for further studies, the second presented the negative aspects. Given the above, the presence ageism and its consequences for the health of the elderly is evident. Finally, studies point to educational strategies on ageism and aging as a way to combat this prejudice.

Keywords: Ageism; Health Care; Aging; Old Man; Preconception.

\section{INTRODUÇÃO}

O século XXI é marcado por um fenômeno a nível mundial: o envelhecimento da sociedade, em especial, do público feminino (Sousa et al., 2018). No Brasil, essa mudança sociodemográfica mostrou uma singularidade, haja vista que, diferente de outros países, ocorreu de forma acelerada em curto espaço de tempo. Nesse sentido, a população 
brasileira deverá atingir, em 2030, o quantitativo de 41,5 milhões de pessoas com idade acima de 64 anos. Essa situação acarretou em novas demandas aos órgãos públicos, à sociedade e aos serviços de saúde. Envelhecer é um processo dinâmico, natural e inevitável, sendo influenciado por diferentes fatores ambientais, sociais e genéticos (Castro et al., 2018).

O processo normal de envelhecimento é denominado senescência, sendo inerente à vida. O patológico é definido como senilidade, este envolve em menor ou maior grau a presença de doenças crônicas (Oliveira \& Corradi, 2018). Apesar dessa ambivalência, é comum uma homogeneização do contingente sênior como portador de doenças crônicas, incapacidades físicas e alterações cognitivas. Essa visão errônea da velhice e do velho são exemplificadas por crenças e comportamentos negativos. O preconceito e a discriminação de idade são inerentes às diferentes culturas, sociedades e tempo. No entanto, somente em 1969 o conceito de ageism (ageísmo) foi cunhado e descrito, por Robert N. Butler, psiquiatra e gerontologista, como um processo de discriminação e de estereótipos de idade (Butler, 1969). Nos dias atuais, decorrente do aumento do número de idosos e da necessidade de compreender o envelhecimento como fenômeno social, pesquisas sobre o ageísmo são pertinentes, no entanto, estudos investigando essa temática ainda são escassos, quando comparados ao racismo e sexismo. Consoante Butler (2008), o ageísmo ocorre em três principais contextos: meio social, local de trabalho e no sistema de saúde.

O presente estudo tem como foco a presença do ageísmo nos ambientes de saúde, principalmente no momento da assistência à saúde. A relação entre ageísmo e saúde evidenciando sua influência negativa em várias facetas do tratamento prestado, desempenho físico, cognitivo, participação social e como variável preditora de doenças na população idosa, encontra-se bem estabelecida na literatura, sendo sustentada por estudos experimentais, longitudinais e meta-analises (Rucker et al., 2018). Nessa perspectiva, o cuidado com o paciente idoso deve ser efetuado de forma integrada, acolhendo o idoso em todas as suas singularidades (Veras \& Oliveira, 2018). Em contrapartida, o ageísmo é citado como um dos principais desafios enfrentados pela terceira idade na busca por atendimento (Walker, Dissanayaka, Scott, Manchha, \& Pachana, 2019). A partir dessa problemática a seguinte questão de pesquisa emergiu: "Como o ageísmo se expressa e é investigado na assistência à saúde do idoso e quais os desfechos apresentados pela literatura?" Diante disso, o objetivo desse estudo é analisar a expressão do ageísmo nos cuidados em saúde, bem como vem sendo investigado pela literatura e seu desfecho para à saúde do idoso. 


\section{METODOLOGIA}

O presente estudo trata de uma revisão sistemática de literatura realizada nas bases de dados: Scientific Electronic Library - SciELO, Literatura Latino-Americana e do Caribe em Ciências da Saúde - LILACS, PsycINFO, Pubmed Central, Web Of Science e Scopus. A busca nos periódicos abrangeu artigos em inglês e português, sendo selecionados artigos publicados no período de janeiro de 2010 a julho de 2019. No tocante ao foco dos estudos, pesquisas que investigassem a presença do ageísmo nos serviços de saúde, as atitudes dos profissionais e estudantes de saúde, as percepções dos idosos sobre os serviços prestados por profissionais de saúde. Foram incluídos artigos que utilizassem um dos seguintes termos: "discriminação de idade", "preconceito etário", "ageísmo", "estereótipos de idade" e seus correspondentes em inglês "ageism", "ageist", "old stereotypes" nos seus títulos, resumos e/ou objetivos. Sendo excluídos estudos que apesar de terem como tema central o ageísmo, não tivessem como contexto a assistência à saúde da população idosa.

Os termos de busca foram estabelecidos conforme o objetivo e as questões norteadoras, sendo obtidos a partir de consulta aos Descritores de Ciências da Saúde (decs.bvs.br) os termos: "Ageísmo", "Idoso" e "Assistência à saúde" e seus correspondentes em inglês "Ageism", "Aged" e "Healthcare".

A busca nas bases de dados foi realizada em agosto de 2019. Sendo realizada por 2 pesquisadores, e um 3 analisou os artigos divergentes. Foram agrupando os termos com operadores booleanos conforme a chave de busca: (Ageísmo AND idoso AND assistência à saúde) e (Ageism AND Aged AND Healthcare). A seleção dos artigos seguiu as recomendações conforme descritas no checklist Preferred Reporting itens for Systematic Reviews and Meta-Analyses - PRISMA que segundo Galvão, Pansani e Harrad (2015) e Moher et al. (2015) que compreende as etapas de identificação do material nas bases de dados, seleção, elegibilidade e Inclusão, como descrito na Figura 1. Inicialmente, foi encontrado um total de 342 artigos. Seguindo com o processo de seleção, foram excluídos os repetidos $(n=43)$ entre as bases de dados. Realizada essa etapa, seguiu-se com a aplicação dos critérios de inclusão e exclusão, sendo eliminados após a leitura dos títulos $(n=141)$ e resumos $(n=57)$, respectivamente. Após a triagem para leitura dos artigos na íntegra, mais 39 estudos foram excluídos por não se adequarem aos critérios. Por fim, 64 estudos constituíram o banco de dados final para análise (Figura1). 


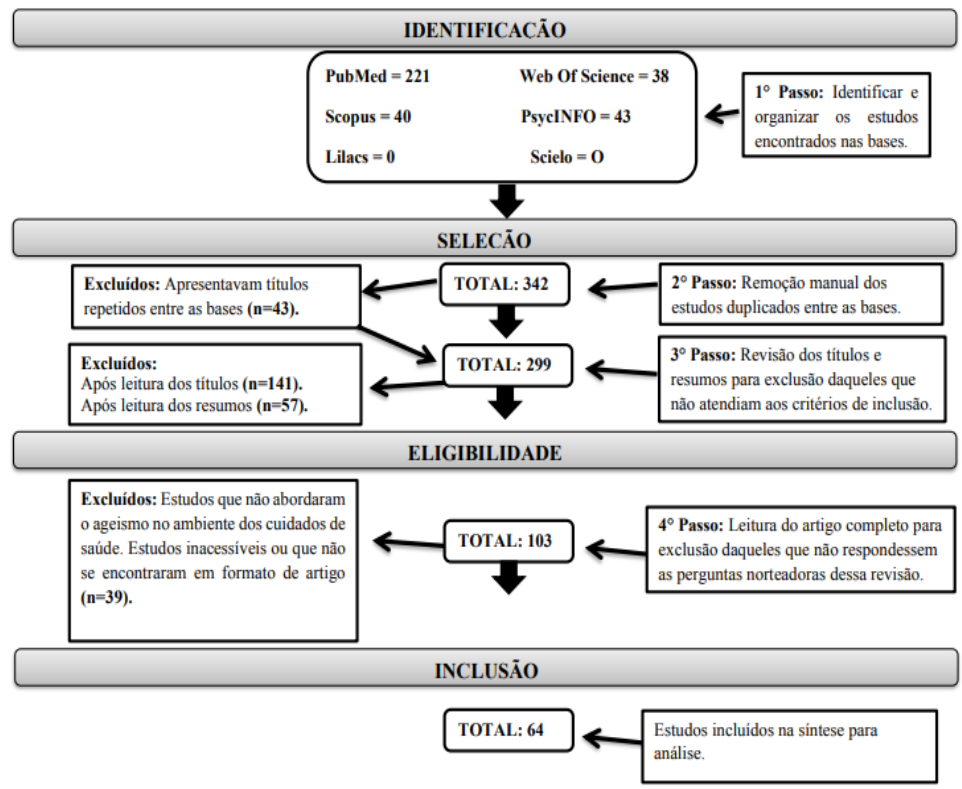

Figura 1. Fluxograma do processo de seleção dos artigos adaptado do PRISMA.

\section{PROCESSAMENTO E ANÁLISE DOS DADOS}

Finalizado o processo de seleção dos artigos, foi realizada a organização do banco de dados em dois corpus textuais monotemáticos. Para criação do primeiro corpus foram extraídos dos artigos as introduções e objetivos. Em estudos teóricos foi considerado como introdução todo o texto até o tópico considerações finais ou conclusão.

O segundo, foi composto pelas conclusões ou considerações finais dos estudos. Posteriormente a criação de ambos os corpus, seguiu-se com um processamento textual dos dados, pelo Iramuteq (Software Interface de $R$ Analyzes Multidimensionais de Textos e Questionários) versão 0,7 alpha 2. Consiste em um software gratuito, de fácil acesso, disponível em vários idiomas, utilizado em diferentes pesquisas qualitativas e possibilita a realização de análises textuais básicas e multivariadas, como a Classificação Hierárquica Descendente (CHD) e similitude (Camargo \& Justo, 2013).

Atrelado a isso, a utilização desse software vem crescendo no Brasil, nos últimos anos, devido às possibilidades da realização de diferentes tipos de análises lexicais, por possuir um rigor estatístico e com isso permitir a realização de análises mais consistentes e fidedignas (Camargo \& Justo, 2013).

Optou-se por analisar os dois arquivos textuais de forma individualizada, por intermédio da CHD. O referido método permite identificar a relação entre os vocabulários e seus contextos 
lexicais, associado ao fato de identificar qual segmento de texto eles estão relacionados. Além disso, por meio de uma Análise Pós-Fatorial são apresentadas as palavras e variáveis relacionadas a cada uma das classes emergentes da CHD (Camargo \& Justo, 2013). A análise da CHD de ambos os corpus resultou em uma retenção de segmentos de texto superior a $75 \%$ dessa forma garantindo o adequado processamento do arquivo textual (Camargo \& Justo, 2013). A classe pode ser entendida como um aglomerado de palavras que compõem um segmento específico. Essas palavras possuem aproximação entre si, e a partir delas pontos chaves do texto podem ser identificados, além disso é possível compreender como esses termos se relacionam entre si (Camargo \& Justo, 2013).

A partir da CHD, o software IRAMUTEQ cria um dendograma que representa a composição e quantitativo lexical de cada classe, bem com a relação entre elas (Camargo \& Justo, 2013). No tocante a composição das classes, somente formas lexicais com frequência superior ao dobro da média de ocorrências associadas a um qui-quadrado $\left(x^{2}\right)$ com valores mais altos $\left(X^{2} \geq 11,52\right)$, ou seja, três vezes o valor mínimo de 3,84 foram selecionadas. Estando descritas no dendograma as palavras, $x^{2}$ e sua frequência de ocorrência por segmento de texto. Estas especificações têm como perspectiva diminuir a margem de falha na associação de cada palavra ou vocábulo com sua classe correspondente.

\section{RESULTADOS}

Em relação ao quantitativo por ano, a análise temporal evidenciou uma baixa produtividade com formação de patamar entre o período de 2010 a 2012 (n:2), seguido de um crescimento ascendente entre 2012 a 2014 (n:9), após esse período o número de publicações se manteve estável com uma média 8,25 publicações entre o período de 2014 a 2017, retomando o crescimento em 2018 (n:16) que alcançou o maior índice nos últimos 10 anos. Uma das possíveis causas do decréscimo aparente no ano de 2019 (n:5) pode ser resultante da busca ter sido realizada até metade do ano em questão.

O corpus 1 "Aspectos teóricos e objetivos" apresentou 47.712 ocorrências com 5440 palavras distintas, alcançando uma média de 8,77 ocorrência por palavra e um aproveitamento de $86,82 \%$ (1192 segmentos de texto). A partir da análise pela CHD, emergiram 2 classes pelo software (Figura2). 


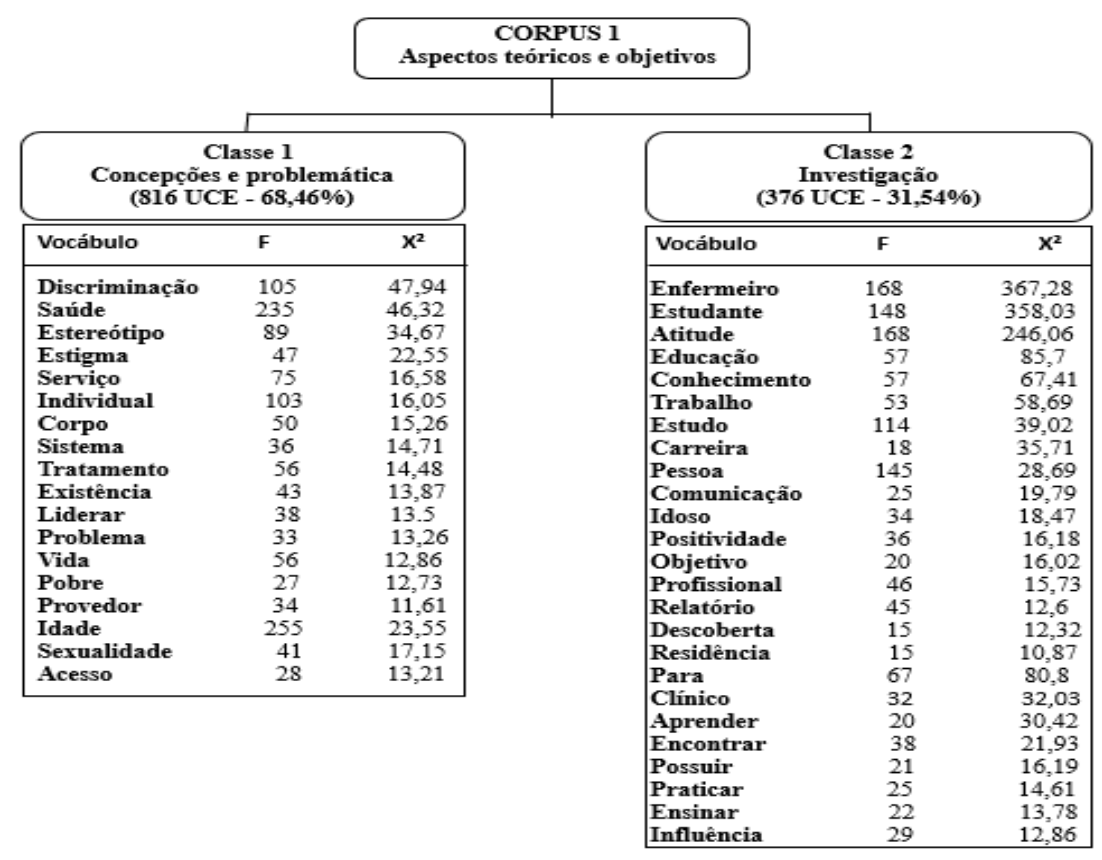

Figura 2. Dendograma do Corpus 1 - Aspectos teóricos e objetivos.

Classe 1 "Concepções e problemática" refere-se aos problemas de pesquisa e a conceitualização do ageísmo no âmbito da saúde. Resultou de um aproveitamento de $68,46 \%$ do corpus com 816 Unidades de contexto elementar (UCE,s). As palavras "Discriminação" "Saúde" "Estereótipo" e "Estigma" tiveram maior expressão. Como por exemplo, nos seguintes segmentos de texto: "Discriminação de idade positiva tem sido usada para resolver algumas desigualdades na saúde, mas a discriminação de idade negativa tem implicações na própria saúde" (Collier \& Foster, 2013). "Ageísmo é um conceito multifatorial que envolve estereotipar idosos em relação a construções sociais que podem levar a estigma, preconceito e comportamento discriminatório" (Banister, 2018). A classe 2 "Investigação" relacionada principalmente com os objetivos dos estudos e o cenário de investigação, com um aproveitamento de $31,54 \%$, os vocábulos com maior destaque foram "Enfermagem" "Estudantes" "Atitudes" e "Educação". Além disso, os valores elevados do qui-quadrado evidenciaram uma forte associação entre as três primeiras palavras e a classe correspondente. A utilização dessas palavras se deu conforme os segmentos de texto a seguir: "Os estudantes de enfermagem atribuíam sua falta de interesse à natureza do trabalho a um medo de lidar com a morte, o sofrimento e dificuldades na comunicação com idosos" (Bahadir-Yilmaz, 2018). "Os enfermeiros tinham atitudes negativas em relação aos idosos, portanto, medidas devem ser tomadas para melhorar as atitudes dos 
enfermeiros em relação aos cuidados com os idosos, especialmente em contextos educacionais em saúde" (Gholamzadeh, Khastavaneh, Khademian \& Ghadakpour, 2018).

O corpus 2 "Aspectos conclusivos" com um aproveitamento de 96,38\%, apresentou 2237 palavras, 1216 segmentos de texto, resultando em 44801 ocorrências, com uma média 20,02 ocorrências por palavra. Decorrente à análise da CHD pelo software emergiram duas classes (Figura 3).

\begin{tabular}{|c|c|c|c|c|c|}
\hline \multicolumn{6}{|c|}{$\begin{array}{c}\text { CORPUS 2 } \\
\text { Aspectos conclusivos }\end{array}$} \\
\hline \multicolumn{3}{|c|}{$\begin{array}{c}\text { Classe 1 } \\
\text { Sugestões } \\
1016 \text { UCE - 86,69\% } \\
\end{array}$} & \multicolumn{3}{|c|}{$\begin{array}{c}\text { Classe 2 } \\
\text { Aspectos negativos } \\
156 \text { UCE - 13,31\% } \\
\end{array}$} \\
\hline Vocábulo & $\mathbf{F}$ & $\mathbf{X} 2$ & Vocábulo & $\mathbf{F}$ & $\mathbf{x} 2$ \\
\hline Atitude & 205 & 38,15 & Acesso & 60 & 325,97 \\
\hline Idade & 246 & 37,77 & Formal & 24 & 159,58 \\
\hline Estudo & 280 & 36,71 & Estrutura & 28 & 156,93 \\
\hline Idoso & 601 & 36,54 & Barreira & 32 & 139,87 \\
\hline Experiência & 176 & 31,8 & Renda & 24 & 130,32 \\
\hline Enfermeiro & 213 & 30,25 & Baixo & 32 & 123,48 \\
\hline Pessoa & 289 & 17,01 & Comunidade & 32 & 112,92 \\
\hline Positivo & 96 & 16,06 & Amplo & 24 & 91,63 \\
\hline Saúde & 175 & 15,02 & Serviço & 40 & 62,57 \\
\hline Desenvolver & 88 & 14,61 & Médico & 32 & 58,4 \\
\hline Sugerir & 84 & 13,89 & Apoio & 32 & 54,3 \\
\hline Compreender & 80 & 13,18 & Sistema & 28 & 43,5 \\
\hline Educação & 80 & 13,18 & Tudo & 24 & 26,65 \\
\hline População & 76 & 12,48 & Resultado & 32 & 17,44 \\
\hline Possuir & 156 & 27,63 & Interior & 44 & 140,00 \\
\hline Estes & 156 & 27.63 & Parecer & 24 & 130,32 \\
\hline Durante & 637 & 26,42 & Poder & 36 & 98,25 \\
\hline Sobre & 224 & 24,38 & Também & 44 & 95,85 \\
\hline $\begin{array}{l}\text { Necessidade } \\
\text { Se }\end{array}$ & $\begin{array}{l}75 \\
72\end{array}$ & $\begin{array}{l}12,3 \\
11,78\end{array}$ & & & \\
\hline
\end{tabular}

Figura 3. Dendograma do Corpus 2 - Aspectos conclusivos.

Classe 1 "Sugestões" apresenta os resultados com sugestões para novos estudos e a importância do conhecimento e educação sobre o envelhecimento e ageísmo como forma de combater esse preconceito, obteve um aproveitamento de $86,69 \%$ e 1016 UCE, s com as seguintes palavras em evidência "Atitude", "Idade", "Estudo" e "Idoso". Conforme exemplificado nos segmentos de texto a seguir: "é necessária pesquisa para examinar as atitudes que os estudantes de enfermagem têm em relação às pessoas idosas. Além disso, é importante determinar se as experiências de aprendizado clínico estão influenciando suas atitudes" Hovey, Dyck, Kim e Reese (2018). "entretanto, este estudo revelou que existe uma correlação entre os valores ocupacionais dos estudantes de enfermagem e suas atitudes em relação ao ageísmo" (Bahadir-Yilmaz, 2018). Classe 2 "Aspectos negativos" relacionado às consequências negativas e dificuldades de combater, obteve um aproveitamento de $13,31 \%$, 156 UCE,s as palavras com maior expressão são "Acesso" "Formal" "Estrutura" "Barreira". 
As palavras são exemplificadas nos seguintes segmentos de texto: "No escopo de idosos frágeis que vivem na comunidade, este estudo destaca que apesar de todas as medidas políticas o acesso a um amplo espectro de serviços de assistência e apoio continua sendo um desafio" (Fret et al., 2019). "Ainda, a necessidade de mais trabalho e - estruturas mais amplas e abordagens - é evidente, dada a dinâmica da mudança de idade" (North \& Fiske, 2012).

\section{DISCUSSÃO}

Os aspectos teóricos e objetivos dos estudos apresentaram como os idosos são vítimas de discriminação em vários contextos socioculturais, resultante de atitudes negativas frente ao envelhecimento e a pessoa idosa, esse cenário favorece o surgimento do ageísmo. Esse tipo de preconceito ocorre de forma recorrente nos serviços de saúde, principalmente nos ambientes de cuidados médicos (Walker et al., 2019). Em comparação com os outros tipos de preconceito o ageísmo é o menos estudado, socialmente aceito, de difícil investigação e camuflado por atitudes pseudo-positivas, em diversões cenários (França, Siqueira-Brito, Valentin, Vasques-Menezes, \& Torres, 2017).

Dentre os vocábulos a palavra "serviço" compreende o ambiente dos cuidados de saúde. Nessa perspectiva, Başer e Cingil (2018) classificam os serviços de saúde voltados aos idosos em três categorias: atendimento institucional, domiciliar e cuidados diários. A palavra "acesso" remete a porta de entrada, acolhimento e localização. Fret et al., (2019) referem-se a acesso como um elo entre o paciente e o sistema de saúde, sendo formado por cinco dimensões (acessibilidade, disponibilidade, acessibilidade geográfica, acomodação e aceitabilidade), atrelado a isso investigou a presença de barreiras que os idosos vivenciam no momento da procura para ter acesso aos serviços formais de saúde.

Nesse sentido, é necessário compreender as possíveis transformações necessárias nos serviços de saúde para promover um acesso igualitário aos idosos de diferentes regiões aos cuidados de saúde (Błachnio \& Buliński, 2019). Nesse contexto, idosos portadores de doenças, como HIV e o Câncer, relataram mais experiências com o estigma social. Atrelado a isso, podem ser alvos de uma dupla estigmatização ou duplo risco (ageísmo e ao preconceito pela doença) (Schroyen et al., 2017). Ainda nesse sentido, resultados empíricos relacionam o ageísmo a presença de doenças crônicas, ou seja, o processo de senilidade (Demarco et al., 2016). A palavra "sexualidade" apresentou nos estudos problematização em diversos aspectos: Invisibilidade nas campanhas de prevenção e promoção sobre saúde 
sexual, discriminação por orientação sexual no ambiente de saúde e abuso sexual (GewirtzMeydan \& Ayalon, 2017). Já a palavra "estudante" teve relação direta com o público mais investigado nos artigos, alcançando o total de 25 artigos desta revisão, sendo $18 \mathrm{com}$ acadêmicos de enfermagem, 5 de medicina e 2 de odontologia. Os acadêmicos de enfermagem apresentaram baixo conhecimento sobre o processo de envelhecimento e não possuem interesse em trabalhar com os idosos (Gholamzadeh et al., 2018). A "atitude" foi a variável comportamental mais estudada, estando presente nos objetivos de 30 artigos desta revisão. Ela é formada por três componentes: cognitivo, afetivo e o comportamental, que se relaciona com as intenções, sendo formado pelos dois construtos anteriores. Uma prevalência de pesquisas estudando principalmente as atitudes tendenciosas relacionadas a idade, por intermédio de questionários, sem levar em consideração o ambiente de cuidados de saúde foi encontrada nesta revisão, somado a isso, poucos estudos investigam os comportamentos discriminatórios de idade, que representam outro componente do ageísmo (Ben-Harush et al., 2017).

Já os aspectos conclusivos das pesquisas mostram, que apesar da existência de estudos avaliando as atitudes dos estudantes e profissionais de saúde no âmbito dos cuidados ao paciente idoso, os resultados encontrados são dicotômicos, divergindo na presença de atitudes negativas e positivas (Wilson, Kurrle \& Wilson, 2018). Além disso, mais pesquisas são necessárias para comprovar se a mudança positiva nas atitudes tem influência nos comportamentos e conseguinte cuidado prestado aos idosos (Leung, Logiudice, Schwarz \& Brandl, 2011). Polat et al., (2014) apesar de identificar a presença de atitudes positivas em seu estudo, reforça a importância em se ter cautela na alocação de recursos em saúde não ser baseada por um viés de idade. Collier e Foster, (2013) identificaram a influência das crenças e valores no cuidado prestado ao idoso por profissionais de saúde. Samra et al., (2015) apontam a existência de diferenças entre crenças e estereótipos sobre idosos hospitalizados e saudáveis, e, que os estudos com foco no idoso paciente ainda são escassos quando comparados a pesquisas com idosos fora do ambiente de saúde. Outro ponto, é a importância da instituição de estratégias educacionais na graduação e nos cursos de especializações, com aulas abordando o envelhecimento e o ageísmo com o objetivo de promover mudanças nas atitudes (Dobrowolska et al., 2017).

Nessa perspectiva, Gholamzadeh et al., (2018) identificaram que um único workshop sobre empatia foi capaz de modificar as atitudes dos acadêmicos de enfermagem sobre idosos. Nesse sentido, as experiências durante a graduação podem refletir de forma positiva e 
negativa nos comportamentos e atitudes dos acadêmicos e profissionais de saúde, diante disso, é salutar um cuidado na forma que os alunos são expostos a ambientes teóricopráticos com os idosos (Banister, 2018). Durante a graduação, os estágios devem ser ambientes de novas experiências e servir de inspiração, resultando em um elo entre teoria e prática no âmbito do envelhecimento. A partir do exposto, uma melhora na conscientização sobre o ageísmo resultaria em uma melhor satisfação dos pacientes e melhores resultados clínicos, os pontos chaves são educar a equipe de saúde e população sobre o fenômeno do envelhecimento e da existência desse preconceito (Karahan et al., 2016). A presença do ageísmo no acesso aos cuidados de saúde apresentou-se como uma barreira a população idosa na busca por atendimento (Fret et al., 2019). Segundo Schroyen et al., (2017b), no momento da explicação do quadro clínico estudantes de medicina e médicos alteram sua fala o que reverbera na informação fornecida ao paciente sobre sua saúde, essa prática evidencia o preconceito durante a comunicação. Levy et al., (2018), aponta outra faceta negativa desse preconceito no âmbito da saúde: o impacto financeiro. Idosos que são vítimas de ageísmo apresentam mais gastos com cuidados médicos e prevalência de doenças crônicas, segundo o referido autor. Diante disso, combater esse preconceito poderá trazer benefício econômico, com diminuição de gastos e otimização da renda familiar, associado a um impacto positivo na situação monetária do país.

\section{CONCLUSÃO}

A partir da utilização do software iramuteq, por intermédio da CHD, foi possível organizar os dados desta revisão sistemática de forma mais precisa, facilitando a interpretação e análise. Em síntese, essa revisão identificou a enfermagem como a área que mais realiza estudos sobre o ageísmo. Os estudantes alcançaram maior prevalência nas pesquisas em comparação aos profissionais, sendo a atitude o determinante comportamental mais investigado. Devido a relevância de estudar esse fenômeno, novas pesquisas que investiguem formas de enfrentamento desse preconceito pelos idosos. Bem como, a implementação e avaliação de estratégias educacionais para estudantes e profissionais de saúde sobre o preconceito de idade e o envelhecimento humano. Uma limitação deste estudo reside na limitação temporal estabelecida na busca, bem como de ter sido realizado até a metade do ano de 2019. 


\section{REFERÊNCIAS}

Ahmadi, P., Alavi, N. M., Gilasi, H. R., Safa, A., \& Aghajani, M. (2019). Developing a Tool for Evaluating Ageism in Nursing Care of Older People in Kashan/Iran. Journal of Cross-Cultural Gerontology, 34(1), $25-33$. https://doi.org/10.1007/s10823-019-09364-w

Bahadir-Yilmaz, E. (2018). The relationship between nursing students' professional values and their attitudes towards the elderly people: A cross-sectional study. Nurse education today, 70, 8-12. https://doi.org/10.1016/j.nedt.2018.08.007

Banister, C. (2018). The effect of ageism on older people and implications for nursing practice. Nursing Older People, 30(5), 34-37. https://doi.org/10.7748/nop.2018.e1056

Başer, G., \& Cingil, D. (2018). Attitudes toward the older persons and ageism attitudes of healthcare assistants working in nursing homes. Turk Geriatri Dergisi, 21(3), 446-455. https://doi.org/10.31086/tjgeri.2018344060

Ben-Harush, A., Shiovitz-Ezra, S., Doron, I., Alon, S., Leibovitz, A., Golander, H., Haron, Y., \& Ayalon, L. (2017). Ageism among physicians, nurses, and social workers: findings from a qualitative study. European Journal of Ageing, 14(1), 39-48. https://doi.org/10.1007/s10433-016-0389-9

Błachnio, A., \& Buliński, L. (2019). Wellbeing and older adults in primary health care in Poland. Annals of Agricultural and Environmental Medicine, 26(1), 55-61. https://doi.org/10.26444/aaem/85711

Butler, R. N. (1969). Age-ism: another form of biogtry. Gerontologist, 9(4), 243-246.

Butler, R.N. (2008). A Revolução da Longevidade: Os Benefícios e Desafios de Viver uma Vida Longa. Nova York: PublicAffairs.

Camargo, B. V., \& Justo, A. M. (2013). IRAMUTEQ: Um software gratuito para análise de dados textuais. Temas Em Psicologia, 21(2), 513-518. https://doi.org/10.9788/tp2013.2-16

Castro, A. P. R. de, Vidal, E. C. F., Saraiva, A. R. B., Arnaldo, S. de M., Borges, A. M. M., \& Almeida, M. I. de. (2018). Promoção da saúde da pessoa idosa: ações realizadas na atenção primária à saúde. Rev. Bras. Geriatr. Gerontol. (Online), 21(2), 155-163. http://www.scielo.br/scielo.php?script=sci_arttext\&pid=S180998232018000200155

Collier, E., \& Foster, C. (2013). Teaching age and discrimination: a life course perspective. Nurse Education in Practice, 14(4), 333-337. https://doi.org/10.1016/j.nepr.2013.12.001

Demarco, R. F., Brennan-Ing, M., Sprague, C., \& Brown, S. M. (2016). Ageism, Aging and HIV: Community Responses to Prevention, Treatment, Care and Support. Interdisciplinary Topics in Gerontology and Geriatrics, 42, 234-239. https://doi.org/10.1159/000448567

Dobrowolska, B., Jędrzejkiewicz, B., Pilewska-Kozak, A., Zarzycka, D., Ślusarska, B., Deluga, A., Kościołek, A., \& Palese, A. (2017). Age discrimination in healthcare institutions perceived by seniors and students. Nursing Ethics, 26(2), 443-459. https://doi.org/10.1177/0969733017718392

França, L. H. de F. P., Siqueira-Brito, A. da R., Valentin, F., Vasques-Menezes, O., \& Torres, C. V. (2017). Ageismo no contexto organizacional: a percepção de trabalhadores brasileiros. Rev. Bras. Geriatr. Gerontol, 20(6), 765-777.

Fret, B., De Donder, L., Lambotte, D., Dury, S., Van der Elst, M., De Witte, N., Switsers, L., Hoens, S., Van Regenmortel, S., \& Verté, D. (2019). Access to care of frail community-dwelling older adults in Belgium: a qualitative study. Primary Health Care Research \& Development, 20. https://doi.org/10.1017/s1463423619000100

Galvão, T. F., Pansani, T. de S. A., \& Harrad, D. (2015). Principais itens para relatar Revisões sistemáticas e Meta-análises: A recomendação PRISMA. Epidemiologia e Serviços de Saúde, 24(2), 335-342. https://doi.org/10.5123/s1679-49742015000200017

Gewirtz-Meydan, A., \& Ayalon, L. (2017). Physicians' response to sexual dysfunction presented by a younger vs. An older adult. International Journal of Geriatric Psychiatry, 32(12), 1476-1483. 
https://doi.org/10.1002/gps.4638

Gholamzadeh, S., Khastavaneh, M., Khademian, Z., \& Ghadakpour, S. (2018). The effects of empathy skills training on nursing students' empathy and attitudes toward elderly people. BMC Medical Education, 18(1), 1-7. https://doi.org/10.1186/s12909-018-1297-9

Hovey, S. L., Dyck, M. J., Kim, M. J., \& Reese, C. (2018). The effect of first clinical assignments on prelicensure nursing students' attitudes toward older people: An observational study. Contemporary Nurse, 54(2), 139149. https://doi.org/10.1080/10376178.2018.1459759

Karahan, F. S., Hamarta, E., \& Karahan, A. Y. (2016). An anthropological contribution about ageism: Attitudes of elder care and nursing students in Turkey towards ageism. Studies on Ethno-Medicine, 10(1), 59-64. https://doi.org/10.1080/09735070.2016.11905472

Leung, S., Logiudice, D., Schwarz, J., \& Brand, C. (2011). Hospital doctors' attitudes towards older people. Internal Medicine Journal, 41(4), 308-314. https://doi.org/10.1111/j.1445-5994.2009.02140.x.

LEVY, B. R., Slade, M.D., Chang, E.S., Kannoth, S., Wang, S.Y. (2020). Ageism Amplifies Cost and Prevalence of Health Conditions. The Gerontologist. 60 (1): 174-181. doi: 10.1093 / geront / gny131.

Moher, D., Shamseer, L., Clarke, M., Ghersi, D., Liberati, A., Petticrew, M., Shekelle, P., Stewart, L., \& PRISMAP. (2015). Evaluation of ASTM Standard Test Method E 2177, 6 Retroreflectivity of Pavement Markings in a Condition of 7 Wetness. Systematic Reviews, January, 1-9. https://doi.org/10.1186/2046-4053-4-1

North, M. S., \& Fiske, S. T. (2012). An inconvenienced youth? Ageism and its potential intergenerational roots. Psychological Bulletin, 138(5), 982-997. https://doi.org/10.1037/a0027843

Oliveira, H. S. B. de, \& Corradi, M. L. G. (2018). Aspectos farmacológicos do idoso: uma revisão integrativa de literatura. Revista de Medicina, 97(2), 165. https://doi.org/10.11606/issn.1679-9836.v97i2p165-176

Potter, G., Clarke, T., Hackett, S., \& Little, M. (2013). Nursing students and geriatric care: The influence of specific knowledge on evolving values, attitudes, and actions. Nurse Education in Practice, 13(5), 449-453. https://doi.org/10.1016/j.nepr.2013.02.007.

Polat, U., Karadag, A., Demir, N. (2014). Nurses' and physicians' perceptions of older people and attitudes towards older people: Ageism in a hospital in Turkey. Contemp Nurse. 48(1):88-97. doi: 10.5172/conu.2014.48.1.88.

Rucker, R., Barlow, P. B., Hartshorn, J., Kaufman, L., Smith, B., Kossioni, A., \& Marchini, L. (2018). Dual institution validation of an ageism scale for dental students. Special Care in Dentistry, 39(1), 28-33. https://doi.org/10.1111/scd.12341

Samra, R., Griffiths, A., Cox T., Conroy, S., Gordon, A., Gladman, J.R. (2015).Medical students' and doctors' attitudes towards older patients and their care in hospital settings: A conceptualisation. Age and Ageing, v. 44, n. 5, p. 776-783. https://doi.org/10.1093/ageing/afv082

Sousa, D., de Jesus, F., Takase Gonçalves, L. H., Paskulin, M., Girard, L., \& Antar Gamba, M. (2018). SOCIODEMOGRAPHIC PROFILE AND SOCIAL SUPPORT OF ELDERLY PERSONS IN PRIMARY CARE. Journal of Nursing UFPE/Revista de Enfermagem UFPE, 12(4).

Schroyen, S., Missotten, P., Jerusalem, G., Van den Akker, M., Buntinx, F., \& Adam, S. (2017). Association between self-perception of aging, view of cancer and health of older patients in oncology: A one-year longitudinal study. BMC Cancer, 17(1), 1-8. https://doi.org/10.1186/s12885-017-3607-8

Veras, R. P., \& Oliveira, M. (2018). Aging in Brazil: The building of a healthcare model. Ciencia e Saude Coletiva, 23(6), 1929-1936. https://doi.org/10.1590/1413-81232018236.04722018

Walker, N., Dissanayaka, N. N., Scott, T., Manchha, A., \& Pachana, N. A. (2019). Shaping attitudes: The association between prior contact with residential aged care and resistance to enter residential aged care. International Journal of Older People Nursing, April, 1-10. https://doi.org/10.1111/opn.12268

Wilson, M. A. G., Kurrle, S. E., \& Wilson, I. (2018). Understanding Australian medical student attitudes towards older people. Australasian Journal on Ageing, 37(2), 93-98. https://doi.org/10.1111/ajag.12495 